\title{
EQUIVALENCES AND CONGRUENCES ON INFINITE CONWAY GAMES *
}

\author{
Furio Honsell ${ }^{1}$, Marina Lenisa $^{1}$ and Rekha Redamalla ${ }^{2}$
}

\begin{abstract}
Taking the view that infinite plays are draws, we study Conway non-terminating games and non-losing strategies. These admit a sharp coalgebraic presentation, where non-terminating games are seen as a final coalgebra and game contructors, such as disjunctive sum, as final morphisms. We have shown, in a previous paper, that Conway's theory of terminating games can be rephrased naturally in terms of game (pre)congruences. Namely, various conceptually independent notions of equivalence can be defined and shown to coincide on Conway's terminating games. These are the equivalence induced by the ordering on surreal numbers, the contextual equivalence determined by observing what player has a winning strategy, Joyal's categorical equivalence, and, for impartial games, the denotational equivalence induced by Grundy semantics. In this paper, we discuss generalizations of such equivalences to non-terminating games and non-losing strategies. The scenario is even more rich and intriguing in this case. In particular, we investigate efficient characterizations of the contextual equivalence, and we introduce a category of fair strategies and a category of fair pairs of strategies, both generalizing Joyal's category of Conway games and winning strategies. Interestingly, the category of fair pairs captures the equivalence defined by Berlekamp, Conway, Guy on loopy games.
\end{abstract}

Mathematics Subject Classification. 68Q55, 91A40, 91A80.

Keywords and phrases. Conway games, non-wellfounded games, coalgebras, equivalences, Joyal's category.

* Work supported by PRIN Project SISTER 20088HXMYN and by the FIRB Project RBIN04M8S8 (both funded by MIUR), and by the ESF Research Networking Programme GAMES 06-RNP-075 PESC.

1 Dipartimento di Matematica e Informatica, Università di Udine, Viale delle Scienze 206, 33100 Udine, Italy. furio.honsell@comune.udine.it; marina.lenisa@uniud.it

2 Birla Science Centre, Adarsh Nagar, 500063 Hyderabad, India. rrekhareddy@yahoo.com 


\section{INTRODUCTION}

The increasing use of games as a convenient metaphor for modeling interactions has spurred the growth of a broad variety of game definitions in Computer Science. In the presentation of games many related concepts are used, e.g. move, position, play, turn, winning condition, payoff function, strategy, etc. None has a unique definition. Usually, some, but not always the same, are taken as primitive, to which the others are reduced. Many more properties need to be specified before the kind of game one is interested in is actually pinned down, e.g.: perfect knowledge, zero-sum, chance, number of players, finiteness, determinacy, etc. All this together with the wide gamut of games arising in real life calls for a unifying foundational approach to games. In [9], we started such a programme using very unbiased foundational tools, namely algebras and coalgebras.

We build upon Conway's notion of game, [5]. It provides an elementary but sufficiently abstract notion of game amenable to a rich algebraic-coalgebraic treatment because of the special role that sums of games play in this theory.

Conway games are combinatorial games, namely no chance 2-player games, the two players being conventionally called left $(\mathrm{L})$ and right $(\mathrm{R})$. Such games have positions, and in any position there are rules which restrict $\mathrm{L}$ to move to any of certain positions, called the left positions, while $\mathrm{R}$ may similarly move only to certain positions, called the right positions. $\mathrm{L}$ and $\mathrm{R}$ move in turn, and the game is of perfect knowledge, i.e. all positions are public to both players. The game ends when one of the players has no move, the other player being the winner. Many games played on boards are combinatorial games, e.g. Nim, Domineering, Go, Chess. Games, like Nim, where for every position both players have the same set of moves, are called impartial. More general games, like Domineering, Go, Chess, where L and R may have different sets of moves are called partisan. Many notions of games, such as those which arise in set theory, in automata theory, or in semantics of programming languages, can be encoded in Conway's format. In [9], we develop Conway's theory of terminating games and winning strategies under an algebraic perspective, and we introduce and study hypergames, i.e. potentially non-terminating games, and non-losing strategies, using coalgebraic methods. Especially in view of applications, potentially infinite, non-terminating interactions are even more important than finite ones. Traditionally, as in the automata-theoretic literature, see e.g. [19], and denotational game semantics, [1], infinite plays are taken to be winning for one of the players. Differently, we take the natural view that all infinite plays are draws: on infinite plays, apparently, there are no losers, because each player can respond indefinitely. This naturally extends the winning condition on Conway games. As far as applications are concerned, this view has received attention in the context of model checking for the $\mu$-calculus, see e.g. [7]. Theoretically, draws are inevitable as soon as one introduces sums of games.

The study of hypergames and non-losing strategies carried out in [9] and in the present paper will be used as a step towards the study of the more general 
framework where infinite plays can be either draws or winning, and general payoff functions are considered, as in the context of e.g. games for Logics.

Hypergames are defined as forming a final coalgebra, and operations on games can be naturally extended to hypergames, by defining them as final morphisms.

Having defined hypergames as a final coalgebra, rather than e.g. graphs, this allows us to abstract superficial features of positions and to reason directly up-to graph bisimilarity. Our approach is justified by the fact that all important properties of games in Conway's setting, e.g. existence of winning/non-losing strategies, are invariant under bisimilarity.

In the present work, we pursue further the investigation started in [9], by focusing on the notions of partial order/equivalence and (pre)congruence. This approach, as we mentioned earlier, allows for a unifying and perspicuous rephrasing of many results in Conway's theory of terminating games and winning strategies. For instance, the fact that a game has a winning strategy for the second player amounts to checking whether it is equivalent to the empty game. In the case of hypergames and non-losing strategies, (pre)congruences suggest the correct generalizations of the results for games. Congruences in games arise in many conceptually independent ways, and, as often happens in semantics, the gist of many results amounts to showing the coincidence of two congruences defined independently.

We have various notions of equivalences, and hence potential congruences with respect to some given game operations:

- The final equivalence induced by the very notion of hypergame, which abstracts superficial features of positions.

- Contextual equivalences obtained by observing the outcome of a game, i.e. which player has a winning strategy, when the game is plugged in particular classes of contexts, in the style of [9]. This definition yields immediately a congruence, which, however, is rather difficult to establish since one is required to consider all possible contexts. Alternate definitions which use only restricted classes of basic contexts are therefore rather valuable.

- Categorical equivalences defined by the existence of suitable strategies, viewed as morphisms, in the style of Joyal's traced monoidal closed category, [13]. This definition allows us to establish equivalence by looking only at the behaviour of a single game.

- Order equivalences defined through an inductively defined order relationship, in the style of Conway's surreal numbers.

- Denotational semantical equivalences, obtained by interpreting games in a subclass of canonical representatives, in the style of Grundy numbers for impartial games, $[8,18]$. As pointed out in [9], in semantical terms, one can say that Grundy numbers provide a fully abstract denotational semantics to impartial games.

All the above equivalences do coincide on Conway games, as shown in [9], but the situation is much more rich and intriguing in the case of hypergames, e.g. the natural relation on hypergames extending Conway's order is not an order on hypergames. 
In the present work, first we introduce and study contextual equivalences on hypergames, obtained by varying the class of contexts and the players for which we observe the existence of a winning strategy. The significance of the resulting equivalence lies in the fact that it captures the extension of Grundy semantics to impartial hypergames. Then we study categorical partial orders/equivalences on hypergames. Since the immediate generalization of Joyal's definition to non-losing strategies does not yield a category, we introduce somewhat stronger categorical equivalences based on the new notions of fair (non-losing) strategy and fair pair of (non-losing) strategies. Both fair strategies and fair pairs give rise to symmetric monoidal categories, and they generalize the category of Conway games, in the sense that this latter is a full subcategory of the above categories. To our knowledge, fair strategies and fair pairs provide the first notions of categories generalizing Joyal's category in the above sense. Interestingly, the categorical equivalence induced on hypergames by fair pairs captures the equivalence introduced in [4], Chapter 11, on loopy games. Finally, we compare the various notions of congruences which we introduce, providing a hierarchy of congruences.

Comparison with related work. The notion of "membership game" introduced in [3] corresponds to the notion of impartial hypergame, where all infinite plays are deemed winning for player II. However, no algebraic operations on games are considered in this setting and no attempt is made to show that indeed all infinite combinatorial games can be modeled by suitably generalizing the notion of membership game.

Equivalences on possibly non-terminating games have been studied in the literature in the context of Logics, e.g. in [16,20], and more recently in [11]. Contrary to our approach, such games are defined as graphs of positions, and structural equivalences on graphs, such as trace equivalences or various bisimilarities are considered. Results of soundness of these structural equivalences with respect to the existence of winning strategies are then proved. Differently, defining games as the elements of a final coalgebra, we directly work up-to bisimilarity of game graphs, and we study coarser notions of equivalences on top of bisimilarity. A notion of equivalence in this spirit, in the context of games for Logics, is the one induced by the preorder defined in [17]. Here a class of games is considered, having a payoff function with values in a partially order set, and a partial order on games is introduced, whereby two games $x, y$ are such that $x \leq y$ if there is a winning strategy for a player, called mediator, in a suitable compound game $\langle x, y\rangle$. There are some analogies between the definition of such a partial order and the categorical orders introduced in the present paper, but the structure of games being different in particular in the way the two players move on the game, the construction carried out in [17], apparently, does not apply to hypergames. Namely, contrary to games arising in Logics, where each position is labeled by exactly one player, i.e. the player whose turn is to move, on Conway games and hypergames, from any position, there can be moves for both players, L and R. This generality is necessary for analyzing many combinatorial games. Complex games, such as multiple-heap Nim and end-games in Go can be conveniently viewed as the disjoint sum of single-heap Nim games 
or more elementary games in Go, respectively. In this kind of sum, at each step, the next player selects any of the component games and makes any legal move in that component, the other components remaining unchanged. The other player can either choose to move in the same component or in a different one. In this way, the alternation of $\mathrm{L}$ and $\mathrm{R}$ in each single component fails. The extra generality of having moves for both players at any position is essential for analyzing complex combinatorial games.

Our categories of hypergames and fair strategies/pairs provide applications of non-terminating games to the semantics of programming languages, alternative to e.g. $[14,15]$, where a category of non-wellfounded games and partial strategies is considered, which subsumes Joyal's category as a subcategory, but not as a full subcategory. Moreover, in our setting the existence of a morphism captures a non-trivial observational equivalence, while in $[14,15]$ this does not appear to have any direct interpretation in terms of winning/non-losing conditions. Finally, in $[14,15]$, and also in [10], games are viewed as graphs, and they are not taken up-to bisimilarity.

Summary. In Section 2, we recall the definitions of hypergames and non-losing strategies together with some fundamental results, and basic operations on hypergames. In Section 3, we introduce the notion of contextual equivalence, and we study its properties and some efficient characterization. In Section 4, we recall the construction of Joyal's category, and we extend this construction in two ways, using non-losing fair strategies or fair pairs. Then we compare the various notions of categorical and contextual equivalences that we have obtained, and we show that fair pairs capture the equivalence defined on loopy games in [4]. In Section 5, a number of open problems and directions for future work are outlined, including possible applications in Computer Science and alternative notions of sum, all of which admit coalgebraic characterizations, and corresponding contextual equivalences. In the Appendix we collect some basic categorical definitions.

\section{HyPERGAMES AND NON-LOSING STRATEGIES}

In this section, we recall the theory of possibly non-terminating games, hypergames, and non-losing strategies developed in [9]. Such games are combinatorial games, where plays can be possibly unlimited in length. These extend the class originally considered by Conway [5], where only games with finite plays are considered, together with winning strategies. On hypergames the notion of winning strategy is replaced by that of non-losing strategy, since we take non terminating plays to be draws.

Combinatorial games are 2-player games, the two players are called left (L) and right $(\mathrm{R})$. Such games have positions, and since we are interested only in the abstract structure of games, we regard any position $x$ as being completely determined by its left and right options. Potentially, both players can move from any position. We shall use the notation $x=\left(X^{L}, X^{R}\right)$, where $X^{L}, X^{R}$ denote the 

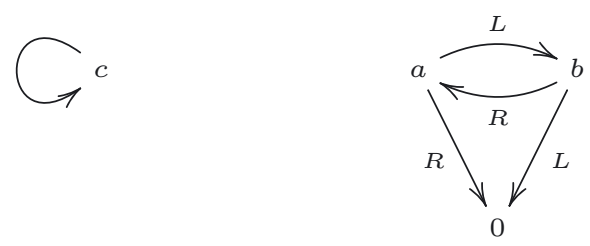

Figure 1. Simple hypergames.

sets of left and right positions, respectively. Games are identified with their initial positions, and they can be represented as the tree of all positions generating from the initial one. $\mathrm{L}$ and $\mathrm{R}$ move in turn, the player who starts the game is called player first, denoted by I, while the one playing second is denoted by II. At the beginning of a game, it is decided whether L or R plays I or II, and from that moment on the players alternate. A game can either terminate when one of the two players does not have any option (the latter being the loser) or the game goes on forever (this being a draw), in the case of infinite games.

Possibly non-terminating combinatorial games can be naturally defined as a final coalgebra on non-wellfounded sets. Non-wellfounded sets (hypersets) are the sets of a universe satisfying the Antifoundation Axiom, see [2,6]. We work in the category Class ${ }^{*}$ of classes of non-wellfounded sets and functional classes, that is proper classes of ordered pairs satisfying the functional condition, (i.e. the second projection is uniquely determined by the first projection $)^{3}$.

Definition 2.1 (hypergames). The set of hypergames $\mathcal{H}$ is the carrier of the final coalgebra $(\mathcal{H}, i d)$ of the functor $F:$ Class $^{*} \rightarrow$ Class $^{*}$, defined by

$$
F(X)=\mathcal{P}(X) \times \mathcal{P}(X)
$$

where $\mathcal{P}(X)$ is the powerset constructor (with usual definition on morphisms).

Games in $\mathcal{H}$ will be denoted by small letters, e.g. $x$, with $x=\left(X^{L}, X^{R}\right)$, and $x^{L}, x^{R}$ will denote generic elements of $X^{L}, X^{R}$.

We denote by $\operatorname{Pos}_{x}$ the set of positions hereditarily reachable from $x$.

Hypergames can be conveniently represented as possibly circular graphs, where nodes represent positions and edges represent L, R options. E.g. the games $a=$ $(\{b\},\{0\})$ and $b=(\{0\},\{a\})$, where $0=(\emptyset, \emptyset)$ denotes the empty game, and $c=(\{c\},\{c\})$, are represented by the graphs in Figure 1. Games like $c$, where $\mathrm{L}$ and $\mathrm{R}$ have the same sets of moves at any position, are represented by unlabeled graphs.

\footnotetext{
${ }^{3}$ Alternatively to classes of sets, we could consider an inaccessible cardinal $\kappa$, and the category whose objects are the sets with hereditary cardinal less than $\kappa$, and whose morphisms are the functions with hereditarily cardinal less than $\kappa$. We recall that the hereditary cardinal of a set is the cardinality of its transitive closure, namely the cardinality of the downward membership tree which has the given set as its root.
} 
By defining hypergames as a final coalgebra, from general results on the theory of coalgebras (see e.g. [12]), we immediately get a coinduction principle for reasoning on them:

Coinduction principle. An $F$-bisimulation on the coalgebra $(\mathcal{H}, i d)$ (hyperbisimulation) is a symmetric relation $\mathcal{R}$ on games such that, for any $x=\left(X^{L}, X^{R}\right)$, $y=\left(Y^{L}, Y^{R}\right)$

$$
x \mathcal{R} y \Longrightarrow\left(\forall x^{L} \in X^{L} \cdot \exists y^{L} \in Y^{L} \cdot x^{L} \mathcal{R} y^{L}\right) \&\left(\forall x^{R} \in X^{R} \cdot \exists y^{R} \in Y^{R} \cdot x^{R} \mathcal{R} y^{R}\right) .
$$

The following coinduction principle holds:

$$
\frac{\mathcal{R} \text { hyperbisimulation } \quad x \mathcal{R} y}{x=y} .
$$

All important notions and constructions on games are invariant with respect to hyperbisimilarity. Moreover, the coalgebraic representation of games naturally induces a minimal representative for each bisimilarity equivalence class.

\subsection{Non-LOSING STRATEGIES}

Let us consider the games $a$ and $b$ of Figure 1 . If $\mathrm{L}$ plays as $\mathrm{I}$ on $b$, then he moves to 0 and he wins since $\mathrm{R}$ has no further move. If $\mathrm{R}$ plays as $\mathrm{I}$ on $b$, then he moves to $a$, then $\mathrm{L}$ moves to $b$ and so on, thus an infinite play is generated. This is a draw. Hence L has a non-losing strategy on $b$ (independently of whether he plays I or II). Symmetrically, R has a non-losing strategy on $a$. Moreover, player $\mathrm{I}$ (independently of whether he plays $\mathrm{L}$ or $\mathrm{R}$ ) has a non-losing strategy both on $a$ and on $b$.

Now let us consider the game $c$ of Figure 1. On this game, both players, L, R, have a non-losing strategy; moreover, also I and II (independently of whether they play L or R) have non-losing strategies. Namely there is only the non-terminating play consisting of infinite $c$ 's.

Strategies over a game can be formalized as partial functions from finite plays to positions. Plays are alternating sequences of positions on the game, starting from the initial position. Non-losing strategies for a given player tell which is the next move of this player, for any play ending with a move of the opponent player.

Here we recall the formal definitions.

Definition 2.2 (plays).

(i) A play on a game $x$ is a (possibly empty) finite or infinite sequence of positions $\pi=x_{1}^{K_{1}} x_{2}^{K_{2}} \ldots$ such that:

- $\forall i . K_{i} \in\{L, R\}$;

- $x=x_{0}$ and $\forall i \geq 0\left(x_{i}^{K_{i}}=\left(X_{i}^{L}, X_{i}^{R}\right) \wedge x_{i+1}^{K_{i+1}} \in X_{i}^{\bar{K}_{i}}\right)$, where $\bar{K}_{i}=$ $\begin{cases}L & \text { if } K_{i}=R \\ R & \text { if } K_{i}=L\end{cases}$ 
We denote by Play $_{x}$ the set plays on $x$, by FPlay $x$ the set of finite plays on $x$, and by $\epsilon$ the empty play.

(ii) A play $\pi$ is winning for player $\mathrm{L}(\mathrm{R})$ if and only if it is finite and it ends with a position $y=\left(Y^{L}, Y^{R}\right)$ where $\mathrm{R}(\mathrm{L})$ is next to move but $Y^{R}=\emptyset\left(Y^{L}=\emptyset\right)$. We denote by WPlay ${ }_{x}^{L}\left(\right.$ WPlay $\left._{x}^{R}\right)$ the set of plays on $x$ winning for $\mathrm{L}(\mathrm{R})$.

(iii) A play $\pi$ is a draw if and only if it is infinite. We denote by DPlay the set $^{2}$ of draw plays.

(iv) A play $\pi$ is non-losing for player $\mathrm{L}(\mathrm{R})$ if and only if it is winning for $\mathrm{L}$ $(\mathrm{R})$ or it is a draw, i.e. we define $\operatorname{NPlay}_{x}^{L}=$ WPlay $_{x}^{L} \cup$ DPlay $_{x}\left(\right.$ NPlay $_{x}^{R}=$ WPlay $_{x}^{R} \cup$ DPlay $\left._{x}\right)$.

In what follows, we denote by:

- FPlay $x_{x}^{L I}$ the set of finite plays on which L acts as player I, and ending with a position where $\mathrm{L}$ is next to move, i.e. FPlay $x_{x}^{L I}=\{\epsilon\} \cup\left\{x_{1}^{K_{1}} \ldots x_{n}^{K_{n}} \in\right.$ FPlay $\left._{x} \mid K_{1}=L \wedge K_{n}=R, n>1\right\}$

- FPlay $x_{x}^{L I I}$ the set of finite plays on which L acts as player II, and ending with a position where L is next to move, i.e. FPlay ${ }_{x}^{L I I}=\left\{x_{1}^{K_{1}} \ldots x_{n}^{K_{n}} \in\right.$ FPlay $_{x} \mid K_{1}=$ $\left.R \wedge K_{n}=R, n \geq 1\right\}$

- similarly we define FPlay ${ }_{x}^{R I}$, FPlay $x_{x}^{R I I}$.

We define:

Definition 2.3 (strategies). Let $x$ be a game.

(i) A strategy $f$ for LI (i.e. L acting as player I) is a partial function $f: F$ Play $_{x}^{L I} \rightarrow$ $\operatorname{Pos}_{x}$ such that, for any $\pi \in$ FPlay $_{x}^{L I}$,

- $f(\pi)=x^{\prime} \Longrightarrow \pi x^{\prime} \in$ FPlay $_{x}$;

- $\exists x^{\prime} . \pi x^{\prime} \in$ FPlay $_{x} \Longrightarrow \pi \in \operatorname{dom}(f)$.

Similarly, one can define strategies for players LII, RI, RII.

(ii) Moreover, we define:

- a strategy for player L is a pair of strategies for LI and LII, $f_{L I} \uplus f_{L I I}$;

- a strategy for player $\mathrm{R}$ is a pair of strategies for RI and RII, $f_{R I} \uplus f_{R I I}$;

- a strategy for player I is a pair of strategies for LI and RI, $f_{L I} \uplus f_{R I}$;

- a strategy for player II is a pair of strategies for LII and RII, $f_{L I I} \uplus f_{R I I}$.

Strategies, as defined above, provide answers (if any) of the given player on all plays ending with a position where the player is next to move. Actually, we are interested only in the behavior of a strategy on those plays which arise when it interacts with (counter)strategies for the opponent player. Formally, we define:

Definition 2.4 (product of strategies). Let $x$ be a game, and $\mathrm{P}$ a player in $\{\mathrm{LI}, \mathrm{LII}, \mathrm{RI}, \mathrm{RII}\}$.

(i) Let $\pi$ be a play on $x$, and $f$ a strategy for $\mathrm{P}$ on $x$. We say that $\pi$ is coherent with $f$ if, for any proper prefix $\pi^{\prime}$ of $\pi$ ending with a position where player $\mathrm{P}$ is next to move,

$$
f\left(\pi^{\prime}\right)=x^{\prime} \Longrightarrow \pi^{\prime} x^{\prime} \text { is a prefix of } \pi \text {. }
$$


(ii) Given a strategy $f$ for $\mathrm{P}$ on $x$, and a counterstrategy $f^{\prime}$, i.e. a strategy for the opponent player, we define the product of $f$ and $f^{\prime}, f * f^{\prime}$, as the unique play coherent with both $f$ and $f^{\prime}$.

As a consequence of the above definitions we have:

Lemma 2.5. The plays coherent with a strategy $f$ are exactly those arising from the product of $f$ with its counterstrategies.

Now we are ready to define non-losing/winning strategies. Intuitively, a strategy is non-losing/winning for a player, if it generates non-losing/winning plays against any possible counterstrategy.

Definition 2.6 (non-losing/winning strategies). Let $x$ be a game, and $\mathrm{P}$ a player in $\{\mathrm{LI}, \mathrm{LII}, \mathrm{RI}, \mathrm{RII}\}$.

(i) A strategy $f$ on $x$ is non-losing for $\mathrm{P}$ if, for any strategy $f^{\prime}$ on $x$ for the opponent player, $f * f^{\prime} \in N$ Play ${ }_{x}^{P}$.

(ii) A strategy $f$ on $x$ is winning for $\mathrm{P}$ if, for any strategy $f^{\prime}$ on $x$ for the opponent player, $f * f^{\prime} \in W P l a y=$.

(iii) A strategy $f_{L I} \uplus f_{L I I}$ for player $\mathrm{L}$ is non-losing/winning if $f_{L I}$ and $f_{L I I}$ are non-losing/winning strategies for LI and LII, respectively. Similarly for players R, I, II.

Notice that on Conway games, where infinite plays do not arise, the notion of non-losing strategy coincides with that of winning strategy.

Intuitively, non-losing strategies are positional, since both the draw condition on infinite plays and the winning condition on finite plays, "no more moves for the next player in the current position", do not depend on the "history", i.e. on the whole sequence of positions, but only on the last position ${ }^{4}$. Namely, one can prove that, for any non-losing/winning strategy on a game $x$, there exists a positional non-losing/winning strategy on $x$ (see [9] for more details).

The following theorem generalizes Conway's determinacy theorem for finite games, on which there exists a winning strategy for exactly one player, L, R, I, II.

Theorem 2.7 (determinacy, [9]).

(i) Any game has a non-losing strategy at least for one of the players L, R, I, II.

(ii) Moreover, either there exists a winning strategy for exactly one of the players $L, R, I, I I$, and there are no non-losing strategies for the other players; or at least two of L, R, I, II have a non-losing strategy. In this latter case either 1 or

\footnotetext{
${ }^{4}$ The notion of positional strategy for hypergames is similar to that of positional strategy for e.g. parity games, on which an analogous result holds, see [7] for the case of three-valued parity games. However, this notion of positional strategy is different from that of history-free strategy used in Game Semantics, where positions are not primitive, and games are defined via sets of moves.
} 
2 holds:

(1) either $L$ or $R$ have a non-losing strategy and either I or II have a non-losing strategy;

(2) all players L, R, I, II have a non-losing strategy.

Remark. Notice that the determinacy theorem does not hold in general for games with more than 2 players, even in the finite case. A counterexample to determinacy is given e.g. by the 3-player Nim game with 2 heaps, each one having 3 elements. One can easily show that no player has a winning strategy on it. Of course we take the reasonable definition that a player who can move when his turn comes cannot be a loser.

\subsection{BASIC GAME OPERATIONS}

We recall the definitions of three basic game operations: grouping, (disjunctive) sum and negation. In the next sections, we will study equivalences which are congruences with respect to such operations.

Grouping. This is the game constructor built-in in the definition of games.

Definition 2.8 (grouping). Let $X^{L}$ and $X^{R}$ be sets of hypergames. Then we can build the hypergame $x=\left(X^{L}, X^{R}\right)$.

Sums. There are various ways in which we can play several different games at once. Each one induces a particular sum game operation. Many different sums have been introduced in [5]. We shall mainly focus on the one which, according to Conway, has been his prime motivation for introducing his theory, namely the disjunctive sum. In this sum, at each step, the next player (L or R) selects any of the component games and makes any legal move in that component, the other components remaining unchanged. The other player can either choose to move in the same component or in a different one. Notice that, in this way, the alternation of players $\mathrm{L}$ and $\mathrm{R}$ in each component fails. The importance of this operation is that it often allows for a natural decomposition of complex games. This is for instance the way a multiple-heap Nim game can be decomposed into the sum of single-heap Nim games, or the way end-games are played in Go.

The generalization to hypergames of the disjunctive sum has been given in [9]:

Definition 2.9 (disjunctive sum). The disjunctive sum of two hypergames is given by the the final morphism $+:\left(\mathcal{H} \times \mathcal{H}, \alpha_{+}\right) \longrightarrow(\mathcal{H}$, id $)$, where the coalgebra morphism $\alpha_{+}: \mathcal{H} \times \mathcal{H} \longrightarrow F(\mathcal{H} \times \mathcal{H})$ is defined by (see Fig. 2):

$\alpha_{+}(x, y)=\left(\left\{\left(x^{L}, y\right) \mid x^{L} \in X^{L}\right\} \cup\left\{\left(x, y^{L}\right) \mid y^{L} \in Y^{L}\right\},\left\{\left(x^{R}, y\right) \mid x^{R} \in X^{R}\right\} \cup\right.$

That is: $x+y=\left(\left\{x^{L}+y \mid x^{L} \in X^{L}\right\} \cup\left\{x+y^{L} \mid y^{L} \in Y^{L}\right\}\right.$, $\left.\left\{\left(x, y^{R}\right) \mid y^{R} \in Y^{R}\right\}\right)$. $\left.\left\{x^{R}+y \mid x^{R} \in X^{R}\right\} \cup\left\{x+y^{R} \mid y^{R} \in Y^{R}\right\}\right)$.

In the following, we will simply refer to disjunctive sum as sum. 

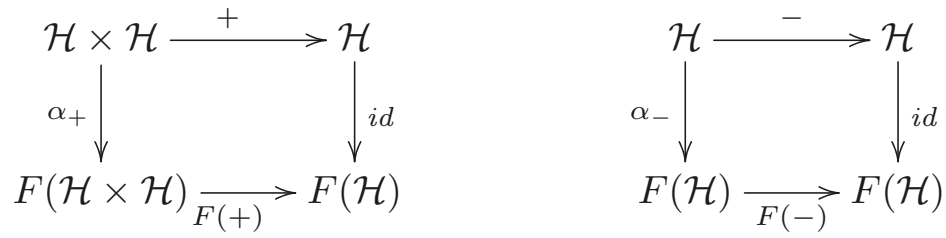

Figure 2. Coalgebraic definitions of sum and negation.

Negation. The negation is a unary game operation, which allows us to build a new game, where the roles of $\mathrm{L}$ and $\mathrm{R}$ are exchanged:

Definition 2.10 (negation). The negation of a hypergame is given by the final morphism $-:\left(\mathcal{H}, \alpha_{-}\right) \longrightarrow(\mathcal{H}$, id $)$, where the coalgebra morphism $\alpha_{-}: \mathcal{H} \longrightarrow$ $F(\mathcal{H})$ is defined by (see Fig. 2):

$\alpha_{-}(x)=\left(\left\{x^{R} \mid x^{R} \in X^{R}\right\},\left\{x^{L} \mid x^{L} \in X^{L}\right\}\right)$.

That is: $-x=\left(\left\{-x^{R} \mid x^{R} \in X^{R}\right\},\left\{-x^{L} \mid x^{L} \in X^{L}\right\}\right)$.

In particular, if $x$ has a non-losing strategy for LI (LII), then $-x$ has a nonlosing strategy for RI (RII), and symmetrically. Taking seriously players L and R and not fixing a priori $\mathrm{L}$ or $\mathrm{R}$ to play first, makes the definition of negation very natural.

Notation. In the following, $x+(-y)$ will be simply denoted by $x-y$.

For the study of properties of sum and negation we refer to [9]. In the following, we study equivalences on games which are congruences with respect to the above basic game operations.

\section{Contextual equivalence}

In this section, we introduce a notion of contextual equivalence on hypergames arising from checking the existence of non-losing strategies in contexts built from the basic game operations introduced in Section 2.2. Then we study a broad spectrum of alternative characterizations of such contextual equivalence, by varying the class of contexts and the players for which we observe the existence of a strategy. A more efficient and practical characterization is also provided, based on the distinction between "well-behaved" games, namely those whose negation is their categorical dual, and those for which this is not the case. This characterization generalizes the one introduced in [9] for impartial hypergames.

Definition 3.1 (equideterminacy). Let $x, y$ be hypergames. We say that $x$ and $y$ are equideterminate (have the same outcome), denoted by $x \Uparrow y$, whenever $x$ has a $\mathrm{L}(\mathrm{R}, \mathrm{I}, \mathrm{II})$ non-losing strategy if and only if $y$ has a L (R, I, II) non-losing strategy. 
We consider the contextual equivalence where contexts are induced by the basic game operations, i.e. grouping, disjunctive sum, and negation:

Definition 3.2 (contextual equivalence).

(i) Let us consider the following class of contexts on hypergames:

$C[]::=[]\left|\left(\{C[]\} \cup\left\{z_{i}\right\}_{i \in I},\left\{z_{j}\right\}_{j \in J}\right)\right|\left(\left\{z_{j}\right\}_{j \in J},\{C[]\} \cup\left\{z_{i}\right\}_{i \in I}\right)|C[]+x|$ $x+C[] \mid-C[]$

for $I, J$ possibly infinite sets of indexes, $z_{i}, z_{j}, x$ hypergames.

(ii) Let $\approx$ be the contextual equivalence on games defined by:

$$
x \approx y \Longleftrightarrow \forall C[] . C[x] \Uparrow C[y] .
$$

Notice that, in the above definition, contexts are taken to be 1-holed (linear). Multi-holed contexts could be equivalently considered, the induced notion of equivalence being the same. This can be easily proved using transitivity of $\approx$.

The contextual equivalence of Definition 3.2 can be proved to be the greatest equivalence refining equideterminacy, which is a congruence with respect to basic game operations; on impartial hypergames, it coincides with the Grundy semantics (see [9] for more details).

Proposition 3.3. The contextual equivalence $\approx$ is the greatest congruence with respect to game operations, which refines equideterminacy.

Proof. By definition, $\approx$ refines equideterminacy, i.e. $x \approx y \Rightarrow x \mathbb{1} y$, and $\approx$ is a congruence, i.e. $x \approx y \Rightarrow \forall C[] . C[x] \approx C[y]$. Now assume that $\simeq$ is a congruence which refines equideterminacy. If $x \simeq y$, then $\forall C[] . C[x] \simeq C[y]$, since $\simeq$ is a congruence; moreover, since $\simeq$ refines equideterminacy, we have $\forall C[] . C[x] \Uparrow C[y]$, hence $x \approx y$.

In the following, we investigate more efficient characterizations of the contextual equivalence.

\subsection{Simplifying the ClASS OF CONTEXtS}

The class of contexts considered in Definition 3.2 can be substantially simplified, namely we show that additive contexts of the shape $D[]=[]+z$, for $z$ any hypergame, are sufficient.

First, we introduce the following notations: for any player $P \in$ $\{L I, L I I, R I, R I I\}$ and hypergames $x, y$,

- let $x \Downarrow_{P}$ denote the fact that $\mathrm{P}$ has a non-losing strategy on $x$;

- let $x \mathbb{\Downarrow}_{P} y$ denote the fact that $\mathrm{P}$ has a non-losing strategy on $x$ if and only if $\mathrm{P}$ has a non-losing strategy on $y$.

\section{Proposition 3.4.}

$$
x \approx y \Longleftrightarrow \forall D[] \cdot D[x] \mathbb{} D[y],
$$

where $D[]$ ranges over contexts of the shape [ ] $+z$, for $z$ any hypergame. 
Proof. Let $\approx^{-}$be the contextual equivalence induced by the class of contexts obtained by eliminating grouping and negation contexts from Definition 3.2 (i). We show that $\approx=\approx^{-}$; then the thesis will follow from commutativity and associativity of sum. Let $x \approx^{-} y$. We prove that for all contexts $C$ [ ] of Definition 3.2(i), for any player $P \in\{L I, L I I, R I, R I I\}, C[x] \mathbb{\Perp}_{P} C[y]$. Assume by contradiction that this is not the case, and let $C[]$ be a minimal context such that $C[x] \mathbb{X}_{P} C[y]$. Assume e.g. $C[x] \Downarrow_{L I}$ but $C[y] \Downarrow_{L I}$, then we consider various cases, according to the shape of $C[]$.

If $C[]=\left(\left\{C_{1}[]\right\} \cup\left\{z_{i}\right\}_{i \in I},\left\{z_{j}\right\}_{j \in J}\right)$, then, according to the non-losing strategy for LI on $C[x]$, L will move to $z_{i}$ or $z_{j}$ or $C_{1}[x]$. If L moves to $z_{i}$ or $z_{j}$, then trivially LI has also a non-losing strategy on $C[y]$. If L moves to $C_{1}[x]$, then $C_{1}[x] \Downarrow_{L I I}$, but $C_{1}[y] \Downarrow_{L I I}$, otherwise $C[y] \Downarrow_{L I}$. But $C_{1}[]$ is a context smaller than $C[]$. Contradiction.

If $C[]=C_{1}[]+z$, with $C_{1}[]$ not having a + operator at the top level, then, according to the non-losing strategy for $\mathrm{LI}$ on $C[x], \mathrm{L}$ will move on $z$ or $C_{1}[x]$. If $\mathrm{L}$ moves on $z$, then he can also perform the same move on $C[y]$, and the game goes on in the same way on $C[x]$ and $C[y]$ until $\mathrm{L}$ or $\mathrm{R}$ moves on $C_{1}[x]$. Since $C_{1}[]$ is not additive, by the shape of $C_{1}[]$, this move cannot be on $x$. Thus this last move brings to $C_{2}[x]$ for some context $C_{2}[]$ smaller than $C[]$, and $C_{2}[x] \Downarrow_{L H}$, while $C_{2}[y] \Downarrow_{L H}$, for $H \in\{I, I I\}$. Contradiction.

If $C[]=-C_{1}[]$, then $C_{1}[x] \Downarrow_{R I}$, while $C_{1}[x] \Downarrow_{R I}$, with $C_{1}[]$ a context smaller than $C[]$. Contradiction.

A further simplification of the class of contexts is obtained by observing that any $z$ having non-losing strategies for all players (L, R, I, II) behaves as a "black hole", that is, for any $x, x+z$ has non-losing strategies for all players. Hence "black holes" do not discriminate more than other additive contexts, and they can be avoided.

\subsection{Simplifying the Class OF STRATEgieS}

One may wonder whether it is sufficient to check the outcome of the games only with respect to the existence of strategies for some player. In this respect, we define:

Definition 3.5. Let $P \in\{L I, L I I, R I, R I I\}$.

(i) The equivalence $\approx_{P}^{D}$ is defined by

$$
x \approx y \Longleftrightarrow \forall D[] . D[x] \mathbb{\Downarrow}_{P} D[y],
$$

where $D[$ ] ranges over contexts of the shape [ ] $+z$, for any hypergame $z$.

(ii) The equivalence $\approx_{P}^{E}$ is defined by

$$
x \approx y \Longleftrightarrow \forall E[] . E[x] \mathbb{\Perp}_{P} E[y],
$$

where $E[]$ ranges over contexts of the shape [ ] $+z$ and -[]$+z$, for any hypergame $z$. 
One can prove that checking strategies for LII (or RII) is sufficient in $E[$ ]contexts.

\section{Lemma 3.6.}

(i) $\approx_{L I}^{E}=\approx_{R I}^{E}$ and $\approx_{L I I}^{E}=\approx_{R I I}^{E}$;

(ii) $\approx_{L I I}^{D} \subseteq \approx_{L I}^{D}$ and $\approx_{R I I}^{D} \subseteq \approx_{R I}^{D}$.

Proof.

(i) We show $\approx_{L I}^{E}=\approx_{R I}^{E}$. The other part being similar. Assume $x \approx_{L I}^{E} y$ and assume by contradiction $E[x] \Downarrow_{R I}$ and $E[y] \Downarrow_{R I}$ for some context $E[]$. Then we have $-E[x] \Downarrow_{L I}$ and $-E[y] \Downarrow_{L I}$, contradicting $x \approx_{L I}^{E} y$.

(ii) We show $\approx_{L I I}^{D} \subseteq \approx_{L I}^{D}$, the other part being similar. Assume by contradiction $x \approx_{L I I}^{D} y, x \Downarrow_{L I}$, but $y \Downarrow_{L I}$. Let us consider the following game:

Then $x+z \Downarrow_{L I I}$. Namely, if $R$ opens in $z$, then $L$ plays I in $x$ and he has a non-losing strategy; if $R$ opens in $x$, then L plays indefinitely in $z$. However, $y+z \Downarrow_{L I I}$, since if $R$ opens in $z$, then $L$ has no non-losing strategy in $y$.

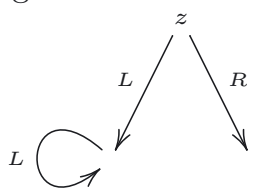

\section{Proposition 3.7.}

$$
\approx_{L I I}^{E}=\approx_{R I I}^{E}=\approx
$$

Proof. Let $x \approx_{L I I}^{E} y$. By Lemma $3.6(\mathrm{i}), x \approx_{R I I}^{E} y$. We are left to show that that also $x \approx_{L I}^{E} y$ and $x \approx_{R I}^{E} y$. From $x \approx_{L I I}^{E} y$ and $x \approx_{R I I}^{E} y$, it follows in particular that $x \approx_{L I I}^{D} y$ and $x \approx_{R I I}^{D} y$. Hence, by Lemma 3.6 (ii), $x \approx_{L I}^{D} y$ and $x \approx_{R I}^{D} y$. Now assume by contradiction $x \neq_{R I}^{E} y$, i.e. there exists a context -[]$+z$ such that e.g. $-x+z \Downarrow_{R I}$ while $-y+z \Downarrow_{R I}$. But then $x-z \Downarrow_{L I}$ while $y-z \Downarrow_{L I}$, contradicting $x \approx_{L I}^{D} y$. Similarly if $x \not_{L I}^{E} y$.

Of course, on impartial hypergames, where $\mathrm{L}$ and $\mathrm{R}$ have the same moves, and hence only player I and II can be considered, the negation context is not needed, and we can restrict ourselves to check strategies for player II just in $D[$ ]-contexts. However, on partisan hypergames, we cannot restrict ourselves to consider strategies for LII (or RII) in $D$ [ ]-contexts (see Lem. 3.8 below for some counterexamples).

Moreover, notice that the situation is not symmetric between player I and II, namely if only non-losing strategies for LI (or RI) in the setting of partisan hypergames are considered (or for player I in the setting of impartial hypergames), in order to recover $\approx$, it is necessary to consider the whole class of original contexts $C[$ ] (sum and grouping contexts, in the impartial case). See Lemma 3.8 below for some counterexamples.

\section{Lemma 3.8.}

$(i) \approx_{L I}^{D} \nsubseteq \approx_{L I I}^{D}, \approx_{L I}^{D} \nsubseteq \approx_{R I}^{D}, \approx_{L I}^{D} \nsubseteq \approx_{R I I}^{D} ;$ 

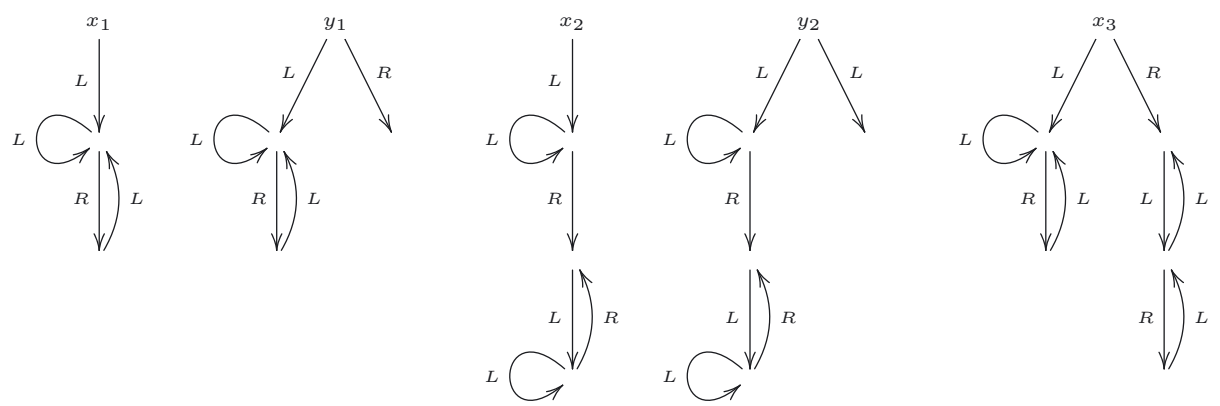

FIGURE 3 .

$($ ii $) \approx_{L I I}^{D} \nsubseteq \approx_{R I}^{D}, \approx_{L I I}^{D} \nsubseteq \approx_{R I I}^{D}$

(iii) the symmetric of the two items above, obtained by exchanging $L$ with $R$.

\section{Proof.}

(i) $\approx_{L I}^{D} \not \approx_{L I I}^{D}, \approx_{R I}^{D}$. Namely, let us consider the games $x_{1}$ and $y_{1}$ in Figure 3 . Then, for all $z, x_{1}+z \Downarrow_{L I}$ and $y_{1}+z \Downarrow_{L I}$; however, $x_{1} \Downarrow_{L I I}$, while $y_{1} \Downarrow_{L I I}$, and $x_{1} \Downarrow_{R I}$, while $y_{1} \Downarrow_{R I}$.

$\approx_{L I}^{D} \nsubseteq \approx_{R I I}^{D}$. Namely, let us consider the games $x_{2}$ and $y_{2}$ in Figure 3 . Then, for all $z, x_{2}+z \Downarrow_{L I}$ and $y_{2}+z \Downarrow_{L I}$; however, $x_{2} \Downarrow_{R I I}$, while $y_{2} \Downarrow_{R I I}$.

(ii) $\approx_{L I I}^{D} \not \subset \approx_{R I}^{D}$. Namely, let us consider the games $x_{1}$ and $x_{3}$ in Figure 3 . Then, for all $z, x_{1}+z \Downarrow_{L I I}$ and $x_{3}+z \Downarrow_{L I I}$; however, $x_{1} \Downarrow_{R I}$, while $x_{3} \Downarrow_{R I}$.

$\approx_{L I I}^{D} \not \approx_{R I I}^{D}$. Namely, let us consider the games $x_{2}$ and $y_{2}$ in Figure 3 . Then, for all $z, x_{2}+z \Downarrow_{L I I}$ and $y_{2}+z \Downarrow_{L I I}$; however, $x_{2} \Downarrow_{R I I}$, while $y_{2} \Downarrow_{R I I}$.

\subsection{An ALternative EFFICIENT CHARACTERIZATION}

The contextual equivalence admits an alternative characterization, based on the distinction between hypergames whose negation is a categorical dual, namely

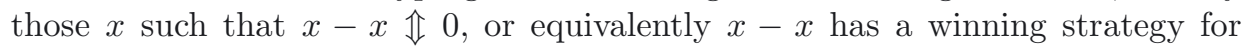
player II, and hypergames such that $x-x \not 0$. The first class strictly includes all Conway games. The hypergames $a, b, c$ of Figure 1 are examples of games in the second class. But there are many hypergames included in the first class, e.g. $w=\left(\left\{w_{1}\right\},\left\{w_{1}\right\}\right)$, where $w_{1}=\left(\left\{w_{1}, 0\right\},\left\{w_{1}, 0\right\}\right)$.

We will show that two hypergames in the first class are contextually equivalent if and only if $x-y \mathbb{1} 0$. This characterization is quite efficient, since we have only to check a single game, i.e. $x-y$. Moreover, it generalizes the characterization of the contextual equivalence on Conway games as the equivalence induced by the partial order on surreal numbers, and the categorical one by Joyal. The games for which $x-x \mathbb{X} 0$ are not well-behaved, since in order to check whether two of them are contextually equivalent, we have to consider, in general, their behavior in all additive contexts (apart from black holes). However, in Section 4, we will provide categorical equivalences for efficiently dealing with all hypergames. 
Definition 3.9. For any hypergame $x$, we define $x \Downarrow$ if and only if $x-x \Uparrow 0$.

Lemma 3.10. If $x \approx y$ and $x \Downarrow$, then $y \Downarrow$ and $x-y \Uparrow 0$.

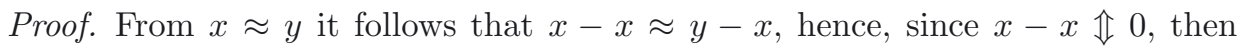
$y-x \Uparrow 0$. Now, from $x \approx y$ it follows that $x-y \approx y-y$, hence $y-x \approx y-y$. Therefore, since $y-x \Uparrow 0$, then also $y-y \Uparrow 0$.

Lemma 3.11. If $x \Uparrow 0$ (i.e. $x$ is a "zero game"), then $\forall z . x+z \Uparrow z$.

Proof. From $x \mathbb{1}$ 0, by definition of $\mathbb{1}$, it follows that $x$ has a winning strategy for II. We have to prove that $x+z \Uparrow z$ for any $z$. Assume $z$ has a non-losing strategy for e.g. L. Then one can easily show that L has a non-losing strategy on $x+z$ as well, whereby L moves in $z$ according to the above non-losing strategy, and responds in $x$ to any move of $\mathrm{R}$ following the winning strategy for II which exists on $x$ by hypothesis. Vice versa assume by contradiction that $\mathrm{L}$ has a non-losing strategy on $x+z$ but no non-losing strategy on $z$. Then, using the Determinacy theorem, one can easily show that RI or RII has a winning strategy on $z$. But then $\mathrm{RI}$ or RII has a winning strategy on $x+z$ as well, whereby $\mathrm{R}$ moves on $z$ according to the winning strategy and plays as II on $x$ according to the winning strategy which exists by hypothesis. But this contradicts the fact that L has a non-losing strategy on $x+z$.

Finally, if we denote by $\mathcal{C}$ the class of "black holes" hypergames, we have:

Theorem 3.12. $x \approx y$ if and only if

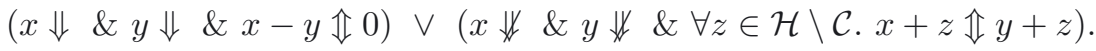

Proof. $(\Rightarrow)$ By Lemma 3.10.

$(\Leftarrow)$ Assume $x \Downarrow, y \Downarrow, x-y \mathbb{\Downarrow} 0$. Then also $y-x \Uparrow 0$. We show that, for any $z$, $x+z \mathbb{\mathbb { }} y+z$. Namely, by Lemma 3.11 , since $y-x \mathbb{1}$, for any $z,(y-x)+(x+z) \mathbb{}$

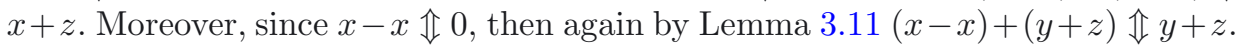
Hence, using transitivity of $\mathbb{\mathbb { }}$, we get $x+z \mathbb{1} y+z$. If $x \notin$ and $y \notin$, then the thesis follows from the observation that black holes do not discriminate more.

The characterization given by the above theorem generalizes the one for impartial hypergames provided in [9]. One may wonder whether the class of contexts on which to test non well-behaved hypergames can be further reduced, e.g. by considering only well-founded $z$ 's, as is the case for impartial hypergames, [9]. The answer is negative. Namely, let us consider the games $u_{L}=\left(\left\{u_{L}\right\}, 0\right), u_{R}=\left(0,\left\{u_{R}\right\}\right)$, and the games $u_{R}^{n}$, for all $n \geq 1$, where $u_{R}^{1}=(0,\{0\}), u_{R}^{n+1}=\left(0,\left\{u_{R}^{n}\right\}\right)$. Then let $x=\left(\left\{u_{L}\right\},\left\{u_{R}\right\}\right)$ and $y=\left(\left\{u_{L}\right\},\left\{0, u_{R}^{1}, u_{R}^{2}, \ldots\right\}\right)$; one can easily check that, for any well-behaved $z, x+z \mathbb{1} y+z$, while $x+u \Downarrow_{R I}$ and $y+u \Downarrow_{R I}$. 


\section{Categorical Characterizations of EQUivalences}

We study generalizations to hypergames of Joyal's category of Conway games and winning strategies. After having observed that the natural extension of Joyal's category to hypergames fails to give a category when all non-losing strategies are considered, we introduce two new symmetric monoidal categories of hypergames: the category of hypergames and fair strategies and that of hypergames and fair pairs of strategies. Quite interestingly, the equivalence induced by this latter category turns out to capture the equivalence introduced in [4], Chapter 11, on loopy games, but it is strictly included in the contextual equivalence.

\subsection{JOYAL'S CATEGORY}

Conway games and winning strategies admit a categorical representation via Joyal's category $\mathcal{Y},[13]$ :

objects:

morphisms:

identity:

composition:

sum:

negation:
Conway games

$f: x \rightarrow y$ winning strategy for LII on $y-x$

copy-cat strategy

via the swivel chair strategy (trace operator)

symmetric monoidal functor

dual object

Theorem 4.1 ([13]). $\mathcal{Y}$ is a compact closed category.

Some comments are in order.

Equivalence. The existence of a winning strategy for LII on $y-x$ corresponds to $y \gtrsim x$, where $\gtrsim$ denotes the partial order on Conway games (see [9] for more details on $\gtrsim)$. Hence the equivalence $\sim$ induced by $\gtrsim$ on Conway games $x, y$ corresponds to the existence of morphisms $f: x \rightarrow y$ and $g: y \rightarrow x$.

Identites. Copy-cat strategies. For any game $x$, there is a copy-cat strategy for LII on $x-x$, according to which player L "copies" at each step the move of player $\mathrm{R}$ in the other component. This corresponds to reflexivity of $\gtrsim$.

Composition. The swivel chair strategy. The closure under composition corresponds to transitivity of $\gtrsim$ and it is guaranteed by the swivel chair strategy. Namely, given strategies for LII, $f$ on $y-x$ and $g$ on $z-y$, a strategy for LII on $z-x$ always exists, obtained by using the "swivel chair", as follows. Assume $\mathrm{R}$ opens on $z-x$, playing either in $z$ or in $-x, e . g$. assume $\mathrm{R}$ opens in $z$. Then $\mathrm{L}$ plays according to the strategy $g$ on $z-y$ : if the $\mathrm{L}$ move according to $g$ is in $z$, then we take this as the $\mathrm{L}$ answer in the strategy on $z-x$; if the $\mathrm{L}$ move according to $f$ is in $-y$ (see Fig. 4), then, using the "swivel chair", we can view this move as an $\mathrm{R}$ move in $y-x$. Now $\mathrm{L}$ has a next move in $y-x$, according to $g$. If this latter move is in $-x$, then we take this as the $\mathrm{L}$ answer in $z-x$; otherwise, if the $\mathrm{L}$ move is in $y$, then we use our swivel chair, viewing this as a move of $\mathrm{R}$ in $-y$ on $z-y$ (see Fig. 4). Hence, according to $f$, $\mathrm{L}$ will have a next move on $z-y$. If this latter is in $z$, then we can take this as the $\mathrm{L}$ move in $z-x$, otherwise we use 


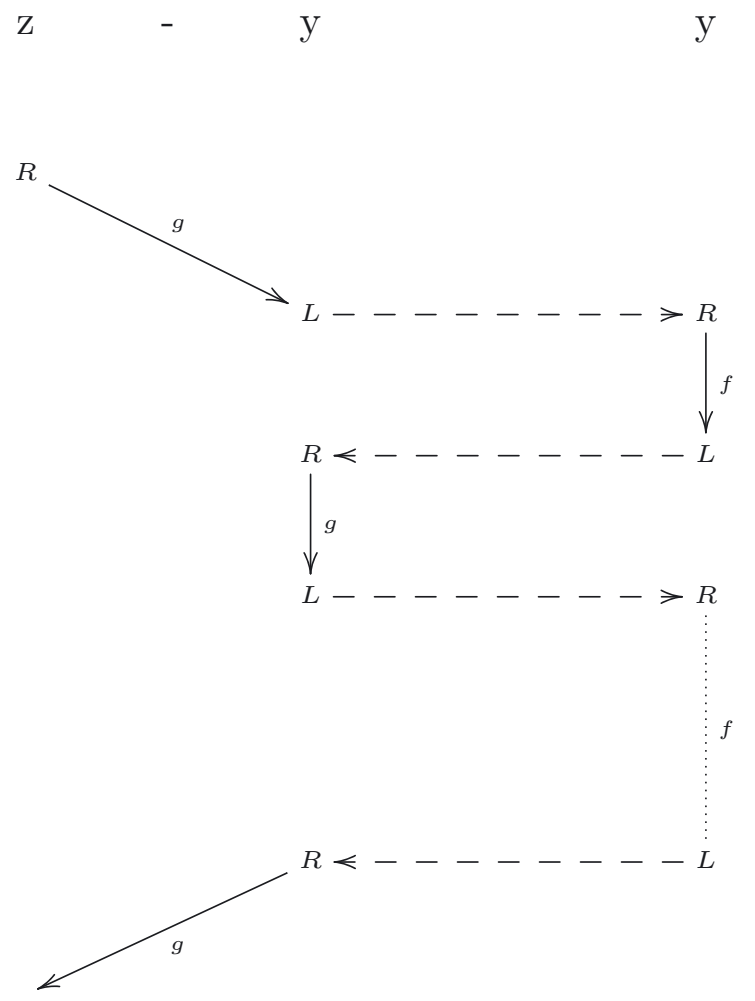

Figure 4. Composition via the swivel chair strategy.

again the swivel chair, and so on. Since both $f$ and $g$ are winning strategies, we are guaranteed that, eventually, the $\mathrm{L}$ move according to $f$ or $g$ will be on $z$ or $-x$. Once we have the $\mathrm{L}$ answer, we go on in the same way, for any possible next $\mathrm{R}$ move.

Closure under composition corresponds to transitivity of Conway's partial order on games $\gtrsim$.

Sum: symmetric monoidal functor. The functoriality of the sum bifunctor follows from the fact that, from any pair of strategies for LII on $x^{\prime}-x$ and $y^{\prime}-y$, one can build a strategy for LII on $x+y-\left(x^{\prime}+y^{\prime}\right)$. This corresponds to the fact that $\gtrsim$ is a precongruence with respect to sum, i.e. $x \gtrsim x^{\prime}$ and $y \gtrsim y^{\prime}$ imply $x+y \gtrsim x^{\prime}+y^{\prime}$.

Negation: dual object. Each object $x$ has a dual, $-x$, whose unit $\left\{\eta_{x}: 0 \rightarrow-x+x\right\}_{x}$ and counit $\left\{\epsilon_{x}: x-x \rightarrow 0\right\}_{x}$ are induced by the copy-cat strategy on $x-x$.

\subsubsection{Extending Joyal's category to infinite games: a first attempt}

The attempt of directly extending Joyal's construction by building a category of games and non-losing strategies fails. Namely, closure under composition fails: applying the swivel chair strategy between the components $y$ and $-y$, as in Figure 4, 
we are not guaranteed to end up in $z-x$. A simple counterexample to closure under composition is the following: let $x=0, y=c, z=d$, where $d=(\emptyset,\{0\})$ and $c$ is the game in Figure 1. Then LII has a non-losing strategy both on $d-c$ and on $c-0$, however LII has no non-losing strategy on $d-0$. Failure of closure under composition of non-losing strategies is related to the fact that the existence of a non-losing strategy for LII on $y-x$ corresponds to $y \unrhd x$, where $\unrhd$ denotes the extension to hypergames of Conway's partial order introduced in [9]. As shown in $[9], \unrhd$ is not transitive. To overcome this shortcoming we introduce categories whose morphisms are a restricted class of non-losing strategies, i.e. fair strategies, or suitable pairs of non-losing strategies, i.e. fair pairs of strategies. Both fair strategies and fair pairs are "well-behaved", in the sense that they are closed under composition, since infinite plays cannot arise in the swivel chair procedure, when such strategies are considered. Both categories are symmetric monoidal and they subsume Joyal's category of Conway games as a full subcategory.

\subsection{A CATEGory of FAir StRAtegies}

Definition 4.2 (fair strategies). Let $x, y$ be hypergames, let $P$ be any player in $\{L I, L I I, R I, R I I\}$.

(i) A non-losing strategy $f$ for $\mathrm{P}$ on $y-x$ is fair if any infinite play coherent with $f$ induces infinite subplays both on $x$ and $y$.

(ii) Let $\gtrsim_{f}$ be the relation defined by: $y \gtrsim_{f} x$ if and only if there is a fair strategy for LII on $y-x$.

(iii) Let $\sim_{f}=\gtrsim_{f} \cap\left(\gtrsim_{f}\right)^{-1}$.

Notice that any winning strategy is fair.

\section{Lemma 4.3.}

(i) $\gtrsim_{f}$ is a partial order;

(ii) $\gtrsim_{f}$ is a precongruence with respect to sum, i.e., for all hypergames $x, y, z$,

$$
x \gtrsim_{f} y \Longrightarrow x+z \gtrsim_{f} y+z .
$$

Proof.

(i) Reflexivity follows from the fact that copy-cat strategies are fair. Transitivity follows from the fact that, if there are fair strategies on $y-x$ and $z-y$, then the swivel chair strategy cannot contain infinite plays, otherwise the strategy on $y-x$ or $z-y$ would not be fair.

(ii) Assume that there is a fair strategy on $y-x$ for LII. Then there is also a fair strategy on $y+z-(x+z)$ for LII , who plays according to the copy-cat strategy when $R$ moves on $z$, and according to the fair strategy on $y-x$ when $R$ moves on $y-x$.

By Lemma 4.3 (i) above, $\sim_{f}$ is an equivalence. Moreover:

Proposition 4.4. The equivalence $\sim_{f}$ refines equideterminacy and it is a congruence with respect to basic game operations. 
Proof. First, we show that, for any context $C[], x \sim_{f} y \Rightarrow C[x] \sim_{f} C[y]$. The proof proceeds by induction on $C[]$ : for sum contexts the thesis follows from Lemma 4.3 (ii) above, for other contexts the thesis follows from the analysis of the shape of contexts and from the definition of $\sim_{f}$.

Now assume $x \sim_{f} y$. We prove that $x \mathbb{\sharp}_{P} y$ for any $P \in\{L I, L I I, R I, R I I\}$. Namely, assume e.g. $x \Downarrow_{L I}$ and $y \Downarrow_{L I}$ (the other cases are dealt with similarly). Then RI has a non-losing strategy on $-x$, and RII has a winning strategy on $y$. Then $\mathrm{R}$ has a non-losing strategy on $y-x$, obtained by playing as $\mathrm{I}$ on $-x$, according to the above non-losing strategy, and as II on $y$, according to the above winning strategy. As a consequence, either LII has no non-losing strategy on $y-x$ because RI wins on $-x$, or we have an infinite subplay on $-x$ but not on $y$, and hence LII has no fair strategy on $y-x$. This contradicts the assumption $x \sim_{f} y$.

As a consequence of Lemma 4.3, we have:

Theorem 4.5. The category $\mathcal{Y}_{f}$ whose objects are hypergames and whose morphisms $f: x \rightarrow y$ are fair strategies for LII on $y-x$ is symmetric monoidal with + as tensor product and the empty game 0 as unit.

The category $\mathcal{Y}_{f}$ provides a generalization of Joyal's category of games. Namely, it is immediate to check that:

Theorem 4.6. The symmetric monoidal category $\mathcal{Y}$ of Conway games and winning strategies is a full subcategory of the symmetric monoidal category $\mathcal{Y}_{f}$.

Notice that negation provides a natural isomorphism $(x \simeq-(-x)$, for any $x)$ on the whole category $\mathcal{Y}_{f}$, but it does not give dual objects. The reason lies in the fact that, for some non-wellfounded games $x$, there are no morphisms in $\mathcal{Y}_{f}$ between $x-x$ and 0. E.g. for $x=c$, where $c$ is the hypergame in Figure 1, and for all hypergames for which it is not the case that $x-x \mathbb{1} 0$. When we consider the subcategory of Conway games, then negation gives us dual objects. Namely, on Conway games, $x-x \Uparrow 0$ and the copy-cat strategy induces natural winning strategies for LII on $(x-x) \rightarrow 0$ and $0 \rightarrow(x-x)$.

\subsection{A CATEgory of FAIR PAirs of Strategies}

The notion of fair pair of strategies is obtained by "splitting" the definition of fair strategy as follows:

Definition 4.7 (fair pairs of strategies). Let $x, y$ be hypergames, let $P \in$ $\{L I, L I I, R I, R I I\}$.

(i) A pair of non-losing strategies $\left\langle f^{+}, f^{-}\right\rangle$for a player $\mathrm{P}$ on $y-x$ is fair if the following two conditions hold:

- for any infinite play $\pi$ coherent with $f^{+}$, if the subplay on $y$ is infinite, then the subplay on $x$ is also infinite; 
- for any infinite play $\pi$ coherent with $f^{-}$. if the subplay on $x$ is infinite, then the subplay on $y$ is also infinite.

(ii) Let $\gtrsim_{p}$ be the relation defined by $y \gtrsim_{p} x$ if and only if there is a fair pair of strategies for LII on $y-x$.

(iii) Let $\sim_{p}=\gtrsim_{p} \cap\left(\gtrsim_{p}\right)^{-1}$.

Notice that any fair strategy $f$ induces a fair pair of strategies $\langle f, f\rangle$. Hence $\sim_{f} \subseteq \sim_{p}$. The converse does not hold, see Section 4.4 below for a counterexample.

\section{Lemma 4.8.}

(i) $\gtrsim_{p}$ is a partial order;

(ii) $\gtrsim_{p}$ is a precongruence with respect to sum, i.e., for all hypergames $x, y, z$,

$$
x \gtrsim_{p} y \Longrightarrow x+z \gtrsim_{p} y+z .
$$

Proof.

(i) Reflexivity follows from the fact that copy-cat strategies are fair, and hence they induce a fair pair. Transitivity follows from the fact that, if there are fair pairs on $y-x$ and $z-y$, then the swivel chair strategy produces a fair pair on $z-x$.

(ii) Assume that there is a fair pair on $y-x$ for LII. Then there is also a fair pair on $y+z-(x+z)$ for LII, who plays according to the the copy-cat strategy when $R$ moves on $z$, and according to the far pair on $y-x$, when $R$ moves on this latter game.

By Lemma $4.8(\mathrm{i})$ above, $\sim_{p}$ is an equivalence. Moreover:

Proposition 4.9. The equivalence $\sim_{p}$ refines equideterminacy, and it is a congruence with respect to basic game operations.

Proof. First, we show that, for any context $C[], x \sim_{p} y \Rightarrow C[x] \sim_{p} C[y]$. The proof proceeds by induction on $C[]$ : for sum contexts the thesis follows from Lemma 4.8 (ii) above, for other contexts the thesis follows from the analysis of the shape of contexts and from the definition of $\sim_{p}$.

Now assume $x \sim_{p} y$. We prove that $x \mathbb{\Downarrow}_{P} y$ for any $P \in\{L I, L I I, R I, R I I\}$. Namely, assume e.g. $x \Downarrow_{L I}$ and $y \Downarrow_{L I}$ (the other cases are dealt with similarly). Then RI has a non-losing strategy on $-x$, and RII has a winning strategy on $y$. Then $\mathrm{R}$ has a non-losing strategy on $y-x$ obtained by playing as $\mathrm{I}$ on $-x$, according to the above non-losing strategy, and as II on $y$, according to the above winning strategy. As a consequence, either LII has no non-losing strategy on $y-x$ because RI wins on $-x$, or the subplay is infinite on $-x$, and hence LII has no fair pair on $y-x$. This contradicts the assumption $x \sim_{p} y$. 
As a consequence of Lemma 4.8 above, we have:

Theorem 4.10. The category $\mathcal{Y}_{p}$ whose objects are hypergames and whose morphisms $f: x \rightarrow y$ are fair pairs for LII on $y-x$ is symmetric monoidal with + as tensor product and the empty game 0 as unit.

The category $\mathcal{Y}_{p}$ generalizes Joyal's category of games:

Theorem 4.11. The symmetric monoidal category $\mathcal{Y}$ of Conway games and winning strategies is a full subcategory of the symmetric monoidal category $\mathcal{Y}_{p}$.

As in the case of $\mathcal{Y}_{f}$, also for $\mathcal{Y}_{p}$ negation does not give dual objects. Namely, for non-wellfounded games $x$, in general, there are no fair pairs of strategies for LII between $x-x$ and 0 .

\subsection{A HIERARCHY OF CONGRUENCES}

The congruences which we have introduced so far give rise to a hierarchy of equivalences. We have already observed that $\sim_{f} \subseteq \sim_{p}$, since any fair strategy gives rise to a fair pair. Moreover, by Propositions 3.3, 4.4 and $4.9, \sim_{f}, \sim_{p} \subseteq \approx$. However, all these congruences are different. Summarizing, we have:

\section{Proposition 4.12.}

$$
\sim_{f} \subsetneq \sim_{p} \subsetneq \approx
$$

In order to prove the above proposition, we are left to show that $\sim_{f} \neq \sim_{p}$ and $\sim_{p} \neq \approx$. These inequalities already hold on impartial hypergames.

In order to show $\sim_{f} \neq \sim_{p}$, we consider the impartial hypergames $a_{1}$ and $b_{1}$ defined by the following graphs. Edges in the game graphs below are not marked, since $\mathrm{L}$ and $\mathrm{R}$ have the same moves.

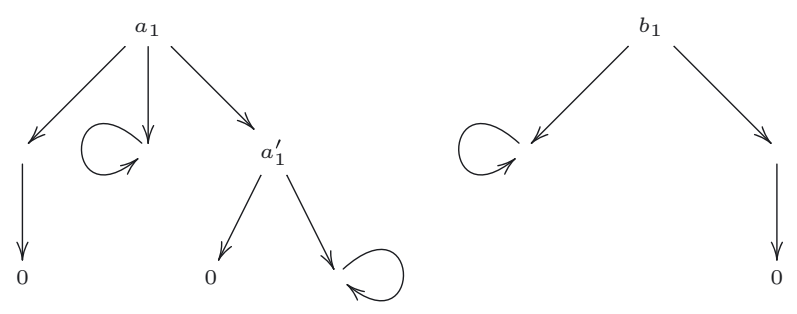

One can check that there is no fair strategy for (L)II on $a_{1}+b_{1}$. Namely, if $\mathrm{R}$ starts moving on $a_{1}$ to $a_{1}^{\prime}$, then, for any subsequent move by $\mathrm{L}, \mathrm{R}$ can force $\mathrm{L}$ to an infinite unfair play. However, one can check, by case inspection, that there is a fair pair for LII on $a_{1}+b_{1}$.

In order to show $\sim_{p} \neq \approx$, we consider the impartial hypergames $a_{2}$ and $b_{2}$ defined by the following graphs. 

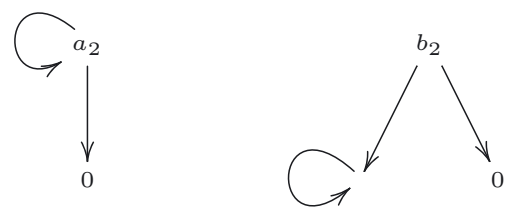

One can prove that $a_{2} \approx b_{2}, e . g$. showing that $a_{2}$ and $b_{2}$ have the same Grundy value (the theory of Grundy values extended to hypergames can be found in [9]). However, (L)II has no fair pair on $a_{2}+b_{2}$, since (R)I can open in $b_{2}$, generating an infinite play on the $b_{2}$ component, forcing the play on $a_{2}$ to end.

In the following, we show that the equivalence $\sim_{p}$ coincides with the equivalence of [4] defined on loopy games.

\subsection{1. $\mathcal{Y}_{p}$ captures the equivalence on loopy games}

Interestingly, the equivalence of fair pairs coincides with the equivalence defined on loopy games in [4], Chapter 11.

Loopy games are possibly non-terminating games on which each infinite play can be either winning for $\mathrm{L}$ or for $\mathrm{R}$ or a draw. In our coalgebraic setting, loopy games can be formalized as follows:

Definition 4.13 (loopy games). A loopy game $\bar{x}=\langle x, \nu\rangle$ is an hypergame $x \in \mathcal{H}$ together with a payoff function $\nu$ such that, for any infinite possibly nonalternating play $\pi$ on $x, \nu(\pi) \in\{L, R, d\}$.

The intended meaning of $\nu(\pi)=L(\nu(\pi)=R)$ is that the play $\pi$ is winning for $\mathrm{L}(\mathrm{R})$, while $\nu(\pi)=d$ means that $\pi$ is a draw.

Notice that, in the definition of loopy games, we require the payoff function to be defined on all possibly non-alternating plays, even if the two players move alternatively on the game. This extra information on non-alternating plays is needed to determine the payoff on the sum of loopy games, since an alternating play on a sum game can induce non-alternating plays on the components. An infinite play on a sum game is then defined as winning for a given player when the infinite plays induced on the components are all winning for that player, otherwise it is a draw.

Definition 4.14 (sum of loopy games). Let $\bar{x}=\left\langle x, \nu_{x}\right\rangle, \bar{y}=\left\langle y, \nu_{y}\right\rangle$ be loopy games. The loopy game sum of $\bar{x}$ and $\bar{y}$ is the game $\bar{x}+\bar{y}=\left\langle x+y, \nu_{x+y}\right\rangle$, where

- $x+y$ is the sum of the hypergames $x$ and $y$; and

- $\nu_{x+y}$ is defined as follows: for any infinite possibly non-alternating play $\pi$ on $x+y$, we denote by $\pi_{x}$ and $\pi_{y}$ the plays induced by $\pi$ on $x$ and $y$, respectively, then

$$
\nu_{x+y}(\pi)=\left\{\begin{array}{l}
L \text { if }\left(\pi_{x} \text { infinite } \Rightarrow \nu_{x}\left(\pi_{x}\right)=L\right) \text { and }\left(\pi_{y} \text { infinite } \Rightarrow \nu_{y}\left(\pi_{y}\right)=L\right) \\
R \text { if }\left(\pi_{x} \text { infinite } \Rightarrow \nu_{x}\left(\pi_{x}\right)=R\right) \text { and }\left(\pi_{y} \text { infinite } \Rightarrow \nu_{y}\left(\pi_{y}\right)=R\right) \\
d \text { otherwise. }
\end{array}\right.
$$


Notice that the subclass of loopy games whose infinite plays are all winning for $\mathrm{L}$ or $\mathrm{R}$ is not closed under the sum. For example, draw plays arise in the sum game $c^{+}+c^{-}$, where $c$ is the game in Figure 1 and $c^{+}, c^{-}$are the loopy games obtained by fixing all infinite plays to be winning for $\mathrm{L}$ and $\mathrm{R}$, respectively. In this sense, draws are inevitable, when the sum of non-terminating games is considered.

The negation of a loopy game is naturally defined as follows:

Definition 4.15 (negation of loopy games). Let $\bar{x}=\left\langle x, \nu_{x}\right\rangle$ be a loopy game. The negation of $\bar{x}$ is the loopy game $-\bar{x}=\left\langle-x, \nu_{-x}\right\rangle$, where $-x$ is the negation of the hypergame $x$, and $\nu_{-x}$ is defined by

$$
\nu_{-x}(\pi)= \begin{cases}L & \text { if } \nu_{x}(\pi)=R \\ R & \text { if } \nu_{x}(\pi)=L \\ d & \text { otherwise. }\end{cases}
$$

Clearly, hypergames are the subclass of loopy games, on which the payoff function on infinite plays always gives $d$.

A strategy on a loopy game $\bar{x}=\left\langle x, \nu_{x}\right\rangle$ is a strategy on the underlying hypergame $x$, as defined in Definition 2.3. Non-losing/winning strategies on loopy games are then defined on the basis of payoff functions:

Definition 4.16 (non-losing/winning strategies on loopy games). Let $\bar{x}=\langle x, \nu\rangle$ be a loopy game, and let $\mathrm{P}$ be a player in $\{K I, K I I\}$, where $K \in\{L, R\}$.

(i) A non-losing strategy for $\mathrm{P}$ on $\bar{x}$ is a strategy $f$ on $x$ such that, for any strategy $f^{\prime}$ on $x$ for the opponent player, $\nu\left(f * f^{\prime}\right) \in\{K, d\}$.

(ii) A winning strategy for $\mathrm{P}$ on $\bar{x}$ is a strategy $f$ on $x$ such that, for any strategy $f^{\prime}$ on $x$ for the opponent player, $\nu\left(f * f^{\prime}\right)=K$.

(iii) A strategy $f_{L I} \uplus f_{L I I}$ for player $\mathrm{L}$ is non-losing/winning if $f_{L I}$ and $f_{L I I}$ are non-losing/winning strategies for LI and LII, respectively. Similarly for players $\mathrm{R}, \mathrm{I}, \mathrm{II}$.

In [4] a relation $\gtrsim_{l}$ on loopy games is defined, in terms of fixed loopy games, that is games on which each infinite play is "fixed" as winning for $\mathrm{L}$ or R, i.e. $\nu(\pi) \in\{L, R\}$.

Definition 4.17 ((in)equality on loopy games, [4]).

(i) Let $x^{*}=\left\langle x, \nu_{x}\right\rangle, y^{*}=\left\langle y, \nu_{y}\right\rangle$ be fixed loopy games. We define

$x^{*} \gtrsim_{f} y^{*}$ if and only if LII has a non-losing strategy on $x^{*}-y^{*}$.

(ii) Let $\bar{x}=\left\langle x, \nu_{x}\right\rangle, \bar{y}=\left\langle y, \nu_{y}\right\rangle$ be loopy games, and let $x^{+}=\left\langle x, \nu_{x^{+}}\right\rangle, y^{+}=$ $\left\langle y, \nu_{y^{+}}\right\rangle, x^{-}=\left\langle x, \nu_{x^{-}}\right\rangle, y^{-}=\left\langle y, \nu_{y^{-}}\right\rangle$denote the fixed loopy games obtained from $\bar{x}$ and $\bar{y}$ by considering all infinite plays which are draws to be winning for $\mathrm{L}$ or $\mathrm{R}$, respectively. We define

$$
\bar{x} \gtrsim_{l} \bar{y} \text { if and only if } x^{+} \gtrsim_{f} y^{+} \wedge x^{-} \gtrsim_{f} y^{-} .
$$


The interesting result is that the categorical relation $\gtrsim_{p}$ determined by fair pairs coincides with the loopy equivalence induced on hypergames, namely:

Lemma 4.18. Let $x, y$ be hypergames, and let $\bar{x}=\left\langle x, \nu_{x}\right\rangle, \bar{y}=\left\langle y, \nu_{y}\right\rangle$ be the corresponding loopy games, where $\nu_{x}(\pi)=\nu_{y}(\pi)=d$ for all infinite possibly nonalternating plays. Then

$$
x \gtrsim_{p} y \text { if and only if } \bar{x} \gtrsim_{l} \bar{y} \text {. }
$$

Proof. The thesis follows from the observation that having a fair pair for LII $\left\langle f^{+}, f^{-}\right\rangle$on the hypergame $x-y$ exactly corresponds to having a pair of nonlosing strategies for LII on the loopy games $x^{+}-y^{+}$and $x^{-}-y^{-}$.

Therefore, we have:

Proposition 4.19. The equivalence induced by $\mathcal{Y}_{p}$ on hypergames coincides with the loopy game equivalence.

More in general, we claim that loopy games can be endowed with the structure of a symmetric monoidal category, when balanced pairs are considered. The induced categorical equivalence captures the loopy equivalence on the whole class of loopy games, thus yielding an alternative elegant characterization of it. We leave it as a future work to suitably extend the above categorical construction to the whole class of loopy games.

\section{FinAL REMARKS AND DIRECTIONS FOR FUTURE WORK}

\section{Applications to the semantics of programming languages}

Games traditionally used in semantics of logics and programming languages (see e.g. [1]) differ from hypergames in some aspects. First of all, in the latter positions are a primitive notion, while in game semantics the notion of move is primitive and positions are defined as alternate sequences of moves. Another key difference is in the sum operation. Conway's disjunctive sum does not necessarily imply that projections in component games are correct plays, i.e. strictly alternating. On the contrary, the sum on semantic games satisfies this condition. This is in accordance with the fact that opponent (R) is fixed as player I and player (L) as II, and legal positions are strictly alternating sequences of moves. A more detailed comparison will appear in a future work.

Categories of Conway-like games, generalizing Joyal's category (in a weak sense) have been recently considered in the setting of game semantics, see e.g. $[14,15]$. However, in these papers categories of non-wellfounded game graphs together with partial strategies are considered, rather than total, i.e. winning or non-losing. Such categories, being compact closed, are useful in the context of semantics. However, 
from the point of view of the induced congruence, which is the main focus of the present paper, these categories are meaningless, since all the games are equated via the empty strategy.

On the other hand, it would be interesting to explore the use of our categories of fair strategies or fair pairs for modeling programming languages. The challenge is to define a trace operator, which apparently lacks on the categories $\mathcal{Y}_{f}$ and $\mathcal{Y}_{p}$.

\section{Applications to model checking}

This is a promising area of applications of hypergames and non-losing strategies. In [7], a three-valued game for the model checking of the $\mu$-calculus has been introduced, together with a notion of non-losing strategy. Such model can be transformed into an equivalent three-valued parity game. It would be interesting to explore the possibility of using hypergames in model checking. This will possibly utilize an encoding of parity games into a suitable extension of hypergames in a world with atoms.

\section{Contextual equivalences vs. categorical equivalences}

The equivalences induced by the categories of fair strategies and fair pairs introduced in this paper is strictly included in the contextual equivalence. The question which arises naturally is whether there is a notion of category capturing the contextual equivalence. On the other hand, we can ask what kind of contextual equivalence is captured by the categories of fair strategies and pairs. In this direction, one can explore contextual equivalences arising from different notions of strategies. An interesting notion to investigate is that of non-terminating strategy, according to which a player wins if he succeeds to force non-termination. Notice that the hypergames $a_{2}$ and $b_{2}$ of Section 4.4 are different with respect to this notion of strategy, since player I has a non-terminating strategy on $b_{2}$, but not on $a_{2}$.

A different approach is that of enlarging the class of contexts in the contextual equivalence, in order to tell apart the hypergames $a_{2}$ and $b_{2}$. A possible operation is one of the conjunctive sums mentioned below.

\section{Other sum operations}

Disjunctive sum is central to the (hyper)game theory. But, as pointed out in [5], there are other ways in which several games can be played at once, e.g.: the selective sum, where at each step the current player selects some components (at least one) and makes a move in each of them; the conjunctive sum, where at each step the current player makes a move in each (non-ended) component.

The above sum operations can be considered in combination with two different ending conditions. With the short ending condition, the game ends when any of the components ends; with the long ending condition, the game ends when all the components are ended.

All the four sums arising above are amenable to coalgebraic definitions. E.g., if for simplicity we work in the setting of impartial hypergames, the selective 
sum with short ending condition is given by the final morphism $\vee$ induced by the coalgebra morphism $\alpha_{\vee}: \mathcal{J} \times \mathcal{J} \longrightarrow \mathcal{P}(\mathcal{J} \times \mathcal{J})$, defined by $\alpha_{\vee}(x, y)=\left\{\left(x^{\prime}, y\right) \mid 0 \neq\right.$ $\left.x^{\prime} \in x\right\} \cup\left\{\left(x, y^{\prime}\right) \mid 0 \neq y^{\prime} \in y\right\} \cup\left\{\left(x^{\prime}, y^{\prime}\right) \mid 0 \neq x^{\prime} \in x \& 0 \neq y^{\prime} \in y\right\} \cup\left\{(0,0) \mid \exists x^{\prime} \in\right.$ $\left.x \cdot x^{\prime}=0 \vee \exists y^{\prime} \in y \cdot y^{\prime}=0\right\}$.

Notice that the two contextually equal games $a_{2}$ and $b_{2}$ of Section 4.4 can be separated by the very simple context [ ] $\Delta\left(\left\{a^{\prime}\right\},\left\{a^{\prime}\right\}\right)$, where $a^{\prime}=(\{0\},\{0\})$ : the first player has a non loosing strategy only when one of the two games fills the context-hole.

It would be interesting to carry out a detailed study of the contextual equivalences induced by classes of contexts involving the various notions of sum. One can prove that the equivalences arising from selective sums are very coarse, namely there are only three equivalence classes: win, lose, draw. The same equivalence should arise from short and long conjunctions. Intuitively, a short conjunction $x_{1} \wedge \ldots \wedge x_{n}$ is won by the player who has the shortest winning strategy on a given component, when the opponent tries to go on as far as possible. If neither of the two players has a winning strategy on any of the components, then this is a draw. Formally, this could be captured by a notion of remoteness of a hypergame, which extends the notion of Steinhaus remoteness for games, [5]. Dually, long conjunction could be dealt with using a notion of suspence, generalizing Steinhaus suspense for games, [5]. Finally, one may ask what kind of equivalences arise by mixing together the various sums. We have only partial results for the moment, even in the impartial case. The first is that the class of all possible contexts induces a congruence which is strictly coarser then hyperbisimilarity. The second result is that the combination of long and short ending conjunctive sum is not the intersection of the two contextual equivalences induced by each of them separately.

\section{Misère}

It would be interesting to explore the equivalences studied in the paper for the misère version of games, i.e. when the winner is the player who does not perform the last move.

Acknowledgements. We would like to thank the anonymous referees for various useful comments, which helped in improving the paper.

\section{Appendix A. CATEgorical Definitions}

Definition A.1 (monoidal category). A category $\mathcal{C}$ is monoidal if

- there is a bifunctor $\otimes: \mathcal{C} \times \mathcal{C} \rightarrow \mathcal{C}$ (tensor product);

- there is a natural isomorphism $\alpha$ with components $\alpha_{A, B, C}:(A \otimes B) \otimes C \cong$ $A \otimes(B \otimes C)$

- there is an object $I$ called the unit or identity;

- there are natural transformations $\lambda$ and $\rho$ with components $\lambda_{A}: I \otimes A \cong A$ 
and $\rho_{A}: A \otimes I \cong A$, satisfying the following commutative diagrams:

- unit coherence law

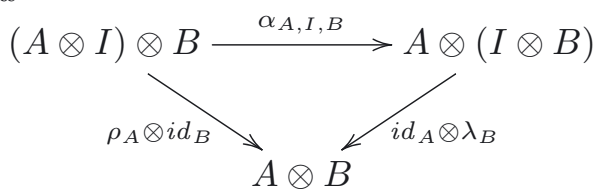

- associativity coherence law

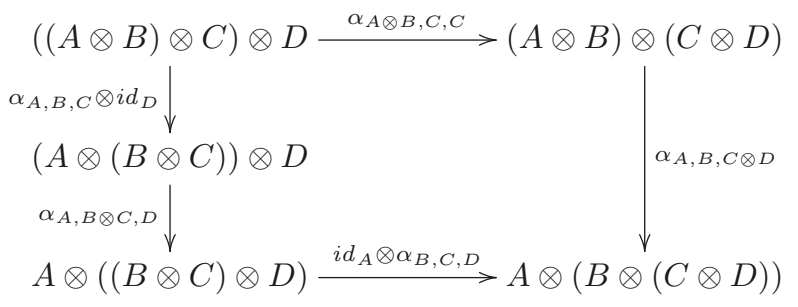

Definition A.2 (symmetric monoidal category). A monoidal category $\mathcal{C}$ with tensor product $\otimes$ is symmetric if there is a natural isomorphism $\sigma$ with components $\sigma_{A, B}: A \otimes B \cong B \otimes A$, satisfying the following commutative diagrams:

- unit coherence for $\sigma$

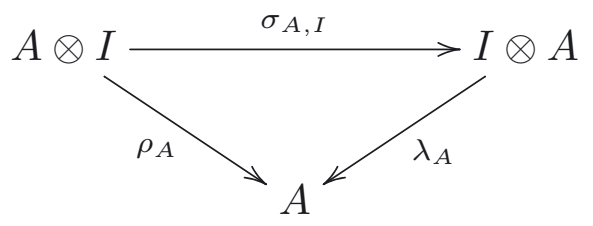

- associativity coherence for $\sigma$

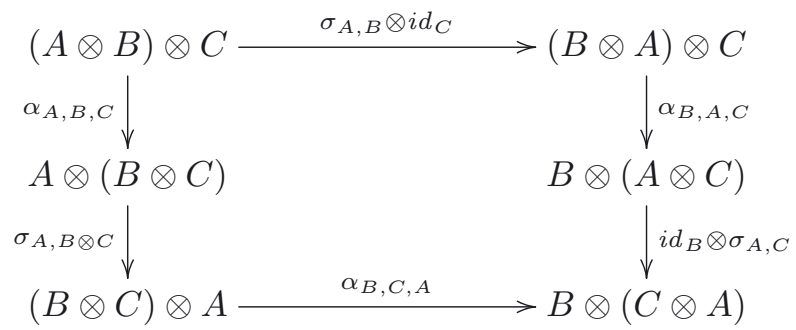

- inverse law

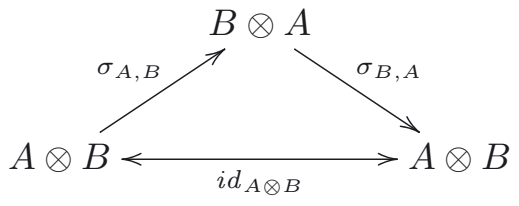


Definition A.3 (dual object). Let $(\mathcal{C}, \otimes, I, \alpha, \lambda, \rho)$ be a symmetric monoidal category. An object $A^{*}$ is the dual of $A$ if there are two morphisms $\eta_{A}: I \rightarrow A^{*} \otimes A$ (unit) and $\epsilon_{A}: A \otimes A^{*} \rightarrow I$ (counit), satisfying the equations:

$\lambda_{A} \circ\left(\epsilon_{A} \otimes i d_{A}\right) \circ \alpha_{A, A^{*}, A^{*}}^{-1} \circ\left(i d_{A} \otimes \eta_{A}\right) \circ \rho_{A}^{-1}=i d_{A}$ and $\rho_{A^{*}} \circ\left(i d_{A^{*}} \otimes \epsilon_{A}\right) \circ \alpha_{A^{*}, A, A^{*}} \circ\left(\eta_{A} \otimes i d_{A^{*}}\right) \circ \lambda_{A^{*}}^{-1}=i d_{A^{*}}$.

Definition A.4 (compact closed category). A symmetric monoidal category $(\mathcal{C}, \otimes, I)$ is compact closed if every object $A$ in $\mathcal{C}$ has a dual object.

Definition A.5 (full subcategory). A subcategory $\mathcal{D}$ of $\mathcal{C}$ is full if, for each pair of objects $A, B$ of $\mathcal{D}, \operatorname{Hom}_{\mathcal{D}}(A, B)=\operatorname{Hom}_{\mathcal{C}}(A, B)$.

\section{REFERENCES}

[1] S. Abramsky and R. Jagadesaan, Games and full completeness for multiplicative linear logic, J. Symb. Log. 59 (1994) 543-574.

[2] P. Aczel, Non-wellfounded sets. CSLI Lecture Notes 14 (1988).

[3] J. Barwise and L. Moss, Vicious Circles. CSLI Lecture Notes 60 (1996).

[4] E. Berlekamp, J. Conway and R. Guy, Winning Ways. Academic Press (1982).

[5] J.H. Conway, On Numbers and Games, 2nd edition (1st edition by Academic Press (1976). AK Peters Ltd. (2001).

[6] M. Forti and F. Honsell, Set-theory with free construction principles. Ann. Scuola Norm. Sup. Pisa Cl. Sci. 10 (1983) 493-522.

[7] O. Grumberg, M. Lange, M. Leucker and S. Shoham, When not losing is better than winning: abstraction and refinement for the full $\mu$-calculus. Inform. Comput. 205 (2007) 1130-1148.

[8] P.M. Grundy, Mathematics and games. Eureka 2 (1939) 6-8.

[9] F. Honsell and M. Lenisa, Conway Games, algebraically and coalgebraically. Log. Meth. Comput. Sci. 7 (2011).

[10] M. Hyland and A. Schalk, Games on Graphs and Sequentially Realizable Functionals, in Proc. of LICS'02. IEEE Computer Science Press (2002) 257-264.

[11] C. Kissig and Y. Venema, Complementation of Coalgebra Automata, in Proc. of CALCO'09. Lect. Notes Comput. Sci. $\mathbf{5 7 2 8}$ (2009) 81-96.

[12] B. Jacobs and J.J.M.M. Rutten, A Tutorial on (Co)algebras and (Co)induction. Bull. of EATCS 62 (1997) 222-259.

[13] A. Joyal, Remarques sur la théorie des jeux à deux personnes. Gaz. Sci. Math. du Québec 1 (1977).

[14] P.L. Curien, H. Herbelin, J.L. Krivine and P.A. Melliès, Categorical semantics of linear logic, in Interactive models of computation and program behaviour. Panoramas et Synthèses, Société Mathématique de France 27 (2009).

[15] P.A. Melliès, N. Tabareau and C. Tasson, An explicit formula for the free exponential modality of linear logic, in Proc. of ICALP'09. Lect. Notes Comput. Sci. 555 (2009).

[16] M. Pauly, From Programs to Games: Invariance and Safety for Bisimulation, in Proc. of CSL'09 (2009) 485-496.

[17] L. Santocanale, Free $\mu$-lattices. J. Pure Appl. Algebra 168 (2002) 227-264.

[18] R. Sprague, Über mathematische kampfspiele. Tohoku Math. J. 41 (1935) 438-444.

[19] W. Thomas, Infinite games and verification, in Proc. of CAV'O2. Lect. Notes Comput. Sci. 2404 (2002) 58-64.

[20] J. van Benthem, Extensive games as process models, J. Log. Lang. Inf. 11 (2002).

Communicated by E. Moggi.

Received December 21, 2010. Accepted January 17, 2012. 